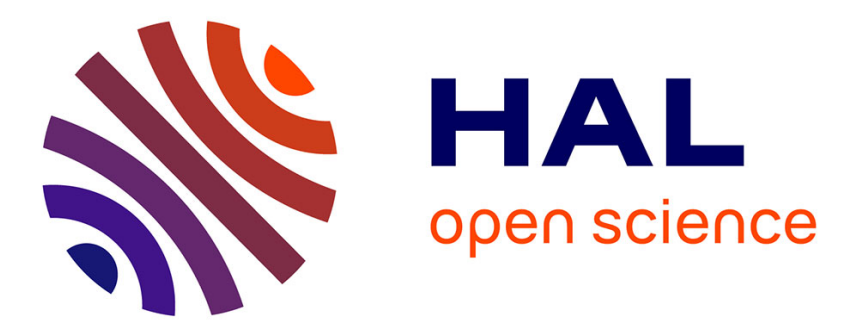

\title{
Theory and Simulations for the Electron Transfer/Ion Transfer Mode of SECM with Electroactive Species Present in Both Liquid Phases
}

Alexander Oleinick, Yun Yu, Michel V Mirkin, Irina Svir, Christian Amatore

\section{To cite this version:}

Alexander Oleinick, Yun Yu, Michel V Mirkin, Irina Svir, Christian Amatore. Theory and Simulations for the Electron Transfer/Ion Transfer Mode of SECM with Electroactive Species Present in Both Liquid Phases. ChemElectroChem, 2018, 10.1002/celc.201800793 . hal-02068112

\section{HAL Id: hal-02068112 \\ https://hal.sorbonne-universite.fr/hal-02068112}

Submitted on 28 Mar 2019

HAL is a multi-disciplinary open access archive for the deposit and dissemination of scientific research documents, whether they are published or not. The documents may come from teaching and research institutions in France or abroad, or from public or private research centers.
L'archive ouverte pluridisciplinaire HAL, est destinée au dépôt et à la diffusion de documents scientifiques de niveau recherche, publiés ou non, émanant des établissements d'enseignement et de recherche français ou étrangers, des laboratoires publics ou privés. 


\title{
Theory and Simulations for the Electron Transfer/Ion Transfer Mode of SECM with Electroactive Species Present in Both Liquid Phases
}

\author{
Alexander OLEINICK, ${ }^{1, *}$ Yun YU, ${ }^{2}$ Michel V. MIRKIN, ${ }^{2}$ Irina SVIR, ${ }^{1}$ Christian AMATORE ${ }^{1,3, *}$ \\ ${ }^{1}$ CNRS UMR 8640 PASTEUR, Ecole Normale Supérieure-PSL Research University, Département de Chimie, Sorbonne \\ Universités -UPMC University Paris 06, 24, rue Lhomond, 75005 Paris, France. \\ 2 Department of Chemistry and Biochemistry, Queens College and the Graduate Center, CUNY, Flushing, NY 11367, \\ USA. \\ ${ }^{3}$ State Key Laboratory of Physical Chemistry of Solid Surfaces, College of Chemistry and Chemical Engineering, Xiamen \\ University, Xiamen, 361005, China.
}

\begin{abstract}
The electron transfer/ion transfer mode of scanning electrochemical microscopy (SECM), in which a nanopipette containing a solvent immiscible with the outer solution is used as a tip to approach a microelectrode substrate, is investigated by simulations in order to analyze the effect of the electroactive species being simultaneously present inside nanopipette and in the external liquid phase. The simulations consider conventional transport modes of the species inside the nanopipette as well as in the bulk solution coupled with their transfer across the liquid-liquid nanointerface supported at the nanopipette tip. The shapes of the simulated approach curves (tip current vs. tip-substrate distance) are highly sensitive to the ratio of initial concentrations in the nanopipette and in the outer solution for a given partition coefficient value providing a direct method to determine its value. The effect of the $1^{\text {st }}$ and $2^{\text {nd }}$ order homogeneous reaction consuming the product electrogenerated at the microelectrode surface onto its collection by the nanopipette is also presented.
\end{abstract}

\section{Keywords:}

electrochemical kinetics, liquid/liquid interface, nanopipette, reactive intermediates, scanning electrochemical microscopy

To whom correspondence should be addressed: Oleksandr.Oliynyk@ens.fr, Christian.Amatore@ens.fr 


\section{Introduction}

Recently, ${ }^{[1]}$ we reported the theory for SECM experiments in which the electroactive species $(A)$ are delivered from a nanopipette to the outer solution via its partitioning across the liquid-liquid nanointerface near an active electrode surface. In that model, the outer solution initially did not contain species $A$. The reduction (or oxidation) of $A$ at the microelectrode surface yielded an ionic product that could be transferred across the liquid-liquid interface supported at the tip of the nanopipette. Under such conditions, the ionic current tracking the collection of the specific ionic product of the electrochemical reaction was shown to drop rather rapidly when the distance, $d$, between the electrode and nanopipette tip exceeded a few times the orifice radius, $R_{\text {pip }}$. It is noteworthy that this sharp decrease exclusively stems from the near thin-layer-cell behavior of the assembly. As a result, the kinetic applications of the method was restricted to situations in which reactions involving the collected ionic species were extremely fast. For example, with a pipette radius in the nanometer range, the kinetic scope of the method was limited to reaction intermediates with lifetimes smaller than ca. $2.5 \mu$ s, i.e., to the timescale too short for many electrochemical mechanisms and whose characterization by cyclic voltammetry (CV) would require ultrafast scan rates in the range $10 \mathrm{kV} / \mathrm{s}-1 \mathrm{MV} / \mathrm{s}^{[4-6]}$ This is certainly of experimental interest owing to the fact that such measurements are performed under steady state without the need for the sophisticated electronics required to compensate ohmic drop and without interference of the charging current. ${ }^{[1,2]}$

Contrariwise, one may conceive a different operating mode using the nanopipette tip as a probe positioned within the diffusion-kinetic layer of the active electrode in order to selectively collect an ionic product of the electrochemical reaction taking place at its surface and measure the lifetime of these species. Simulations of such measurements when the electroactive substrate is initially present in the bulk solution show that this second operation mode is more sensitive to slow kinetics due to the longer diffusion time of the collected species generated from the whole generator electrode to the orifice of the nanopipette. It is also shown that this second operation mode allows direct measurement of the partition coefficient between the solvent contained inside of the nanopipette and the solution in which the electrode performs under the electrified conditions prevailing at the interface.

\section{Model}

In the following, we assume that the disk electrode radius, $R_{\text {disk, }}$, is sufficiently small to reach a diffusioncontrolled steady state without any significant involvement of natural convection. ${ }^{[7]}$ Conversely, $R_{\text {disk }} \gg$ $R_{\text {pip }}$, so that the nanopipette tip faces only a small fraction of the electrode surface at a short separation distance $\left(d / R_{\text {pip }} \rightarrow 0\right)$. Figure 1 represents a sketch of the system investigated together with the definition of the various parameters and coordinates describing the model used in the simulations.

All data reported hereafter are the results of simulations performed using Comsol 5.3a software. ${ }^{[8]}$ The electrochemical reaction at the substrate is:

$$
A \pm n e^{-} \rightleftharpoons \varphi B
$$

where the species $A$ may be present only in the nanopipette, only in the outer solution or simultaneously in both solutions at different relative concentrations. The species $B$ is electrogenerated with a yield $\varphi$ in Eqn (1) at the microelectrode surface and is assumed to be the only product that can be collected at the 
applied potential across the liquid-liquid interface. $B$ may either be chemically stable or undergo a $1^{\text {st }}(m=$ $1)$ or $2^{\text {nd }}$ order $(m=2)$ homogeneous reaction:

$$
m B \rightarrow C
$$

with a rate constant $k_{m}$. Finally, all simulations reported below assume that the transport of $A$ and $B$ occurs under pure diffusion control in both phases and that the transfer kinetics at the interface is much faster than transport processes, i.e. $[A]_{\text {pip }}^{Z^{*}=0}=\kappa[A]_{\text {elec }}^{Z^{*}=0}$, where $\kappa$ is the partition coefficient of $A$, and $[B]_{\text {elec }}^{Z^{*}=0}=$ 0 .

The corresponding physico-mathematical description of the system including kinetics is identical to that described in detail in our previous report ${ }^{[1]}$ except for the fact that, presently, the electrode has a finite radius $R_{\text {disk }}$ (not drawn to scale in Figure 1). Hence, the model is not described here again, but its full description is given in the Supporting Information. In addition, to the best of our knowledge there is no simple analytical solution available for the complex situation investigated here except for its limiting cases, i.e., those describing the behavior of the microelectrode alone or the approach curves for the nanopipette when the bulk outer solution does not initially contain any electroactive material. ${ }^{[1]}$

The approach curves given below represent ionic current flowing through the nanopipette orifice as a function of the tip-substrate separation distance. All simulation results correspond to a single representative set of geometrical and physicochemical parameters, ${ }^{[1,2]}$ i.e. the pipette interior radius, $R_{\text {pip }}=100 \mathrm{~nm}$; the pipette conical angle, $\theta=8^{\circ}$; the ratio of the pipette shaft and orifice radii, $R G=$ $\left(R_{\text {pip }}+g\right) / R_{\text {pip }}=1.5 ; D_{\text {elec }}^{A}=D_{\text {elec }}^{B}=5 \times 10^{-6} \mathrm{~cm}^{2} / \mathrm{s}$ is the diffusion coefficient of $B$ in the external solution; the ratio of diffusion coefficients of species $B$ in the filling and external solution, $\gamma=$ $D_{\text {solv }}^{A} / D_{\text {elec }}^{A}=D_{\text {solv }}^{B} / D_{\text {elec }}^{B}=2 ; R_{\text {disk }}=12.5 \mu \mathrm{m}$. The bulk concentrations in the filling and external solutions, $[A]_{\mathrm{pip}}^{\mathrm{bulk}}$ and $[A]_{\mathrm{elec}}^{\mathrm{bulk}}$, and the partition coefficient value of $A$ between the solvent contained in the pipette and the electrolyte, $\kappa$, are specified in each figure caption below.

a)

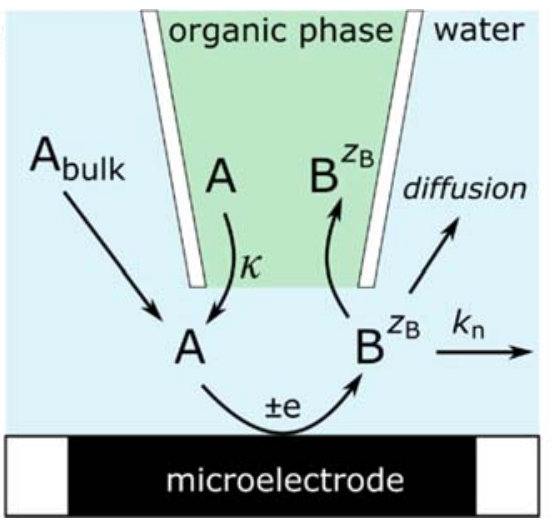

b)

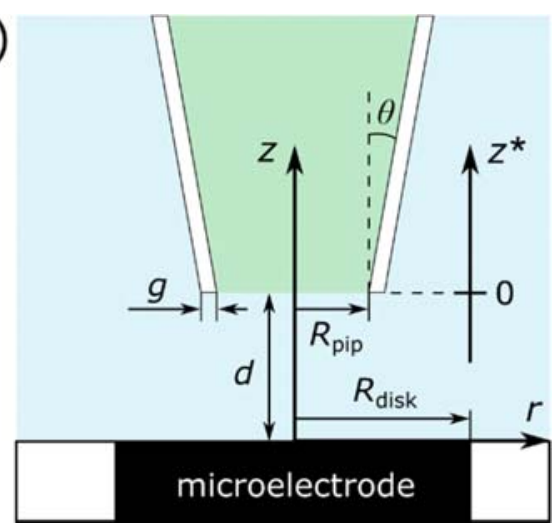

Figure 1. Representation of the nanopipette tip approaching the microelectrode substrate along its symmetry axis (not to scale). (a) Schematic description of the transport, electrochemical and chemical reactions considered in this study. (b) Geometrical parameters of the system: $z=z^{*}+d, \theta$ is the pipette conical angle, $R G=\left(R_{\text {pip }}+g\right) / R_{\text {pip }}=1.5$; other parameters are defined in the text. 
To conclude this section, we wish to define what is meant in this work by the term 'initial conditions'. A priori 'initial conditions' involve a nanopipette loaded with $A$ at a given concentration and immersed into an electrolyte solution containing a different concentration of $A$ (left panels in Figures $2 \mathrm{a}, \mathrm{b}$ ). However, it is clear that equilibration must occur across the nanointerface to reach the condition imposed by the partition coefficient of $A$ between the two media. This, in turn, necessitates the existence of diffusion concentration gradients inside the nanopipette and in the electrolyte (e.g., compare the right panels in Figures $2 \mathrm{a}, \mathrm{b}$ ), so that the above description of 'initial conditions' cannot define the actual status of the system even when no potential is applied to the microelectrode. In this preliminary section we wish to evaluate how the actual state of the system differ from its theoretical 'initial conditions' defined above.

Approximate analytical formulations may be proposed for the steady state concentration gradients that establish on either side of the nanointerface. Indeed, on the one hand, since the nanopipette cone angle $\theta$ is extremely small the nanointerface can be viewed either as a disk of radius $R_{\text {pip }}$ or by narrow spherical cupola delimited on a sphere of much larger radius $R_{\text {sph }}=R_{\text {pip }} / \sin \theta^{[1,9]}$ depending on the phase considered. Therefore, the steady state concentration profile, $[A]_{\text {pip }}\left(z^{*}\right)$, that spontaneously establishes inside the nanopipette can be estimated through reference to the solution for steady state diffusion near a spherical electrode: ${ }^{[10]}$

$$
[A]_{\text {pip }}\left(Z^{*}\right)=[A]_{\text {pip }}^{\text {bulk }}+\left([A]_{\text {pip }}^{Z^{*}=0}-[A]_{\text {pip }}^{\text {bulk }}\right) /\left[1+\left(z^{*} / R_{\text {pip }}\right) \sin \theta\right]
$$

where $z^{*} \geq 0$ is the distance from the nanointerface and $[A]_{\text {pip }}^{Z^{*}=0}$ the concentration at the interface. Similarly, the steady state concentration profile in the electrolyte can be approximated by that created near a disk electrode. Thus, neglecting the fact that $R G$ has not a very large value, one has: ${ }^{[10-12]}$

$$
[A]_{\mathrm{elec}}\left(z^{*}\right)=[A]_{\mathrm{elec}}^{\mathrm{bulk}}+\left([A]_{\mathrm{elec}}^{Z^{*}=0}-[A]_{\mathrm{elec}}^{\mathrm{bulk}}\right) \times \frac{2}{\pi} \arcsin \left[1 / \sqrt{\left(z^{*} / R_{\mathrm{pip}}\right)^{2}+1}\right]
$$

where $z^{*} \leq 0$ is the distance from the nanointerface along the symmetry axis of the system $(r=0)$ and $[A]_{\text {elec }}^{Z^{*}=0}=[A]_{\text {pip }}^{Z^{*}=0} / \kappa$. Equations (3) and (4) indicate that the steady state concentration gradients spontaneously induced by the equilibration process are confined over distances of the order of $R_{\text {pip }}$ on each side of the nanointerface. In other words, the content of the nanopipette and that of the electrolyte are expected to differ from the 'initial conditions' only in the close proximity of the nanointerface.

The above formulations are based on three assumptions: (i) the liquid-liquid nanointerface geometry was simultaneously modelled by a spherical sector (nanopipette side) or a plane disk (outer solution side); (ii) $[A]_{\text {pip }}^{Z^{*}=0}$ and $[A]_{\mathrm{elec}}^{Z^{*}=0}$ were considered to have constant values over the nanointerface; and (iii) $R G \gg 1^{[13]}$ although typical values range between 1.1 and 1.5. Although these approximations were required to propose simple analytical solutions that illustrate the main consequences of the equilibration process it is understood that the predicted values of $[A]_{\text {pip }}^{Z^{*}=0}$ and $[A]_{\text {elec }}^{Z^{*}=0}$ derived through a strict application of Eqns $(3,4)$ must necessarily slightly differ from the real ones. Nonetheless, these estimates were in good agreement with the results of accurate numerical simulations of the system considering a flat disk liquidliquid nanointerface and $R G=1.5$. In Figures $2 \mathrm{a}, \mathrm{b}$, the right panels represent the corresponding simulated concentration maps for species $A$ when $[A]_{\text {pip }}^{\text {bulk }}=0,[A]_{\mathrm{elec}}^{\text {bulk }}=1 \mathrm{mM}$ and $\kappa=50$. The good predictive quality of the spatial dependences in Eqns $(3,4)$ vs. the outcome of accurate numerical simulations is further confirmed by the extremely good agreement between the concentration profiles plotted in Figure 
2c (note that (i) $[A]_{\text {pip }}^{Z^{*}=0}$ and $[A]_{\text {elec }}^{Z^{*}=0}$ values introduced in Eqns (3) and (4) were those determined numerically in order to eliminate the minor biases described above, and (ii) the concentration profile inside the electrolyte was scaled by $\kappa$ for the sake of graphical representation since one has $\kappa[A]_{\mathrm{elec}}^{Z^{*}=0}=[A]_{\mathrm{pip}}^{Z^{*}=0}$ $\left.{ }^{[1]}\right)$.

a)

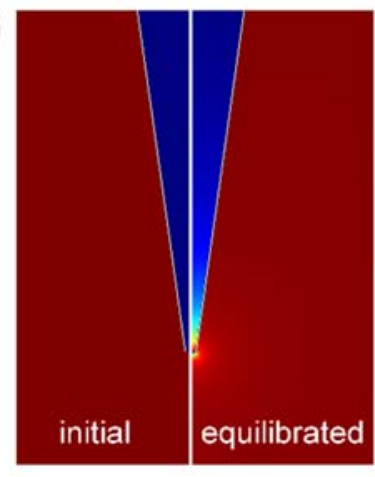

b)

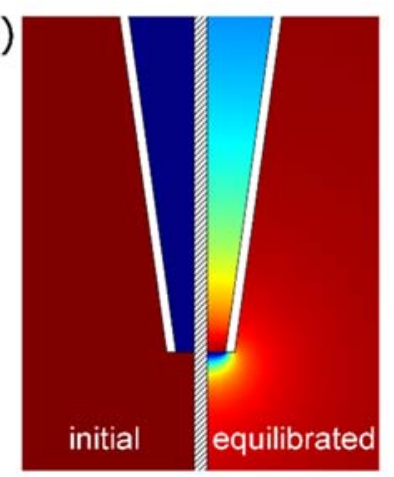

c)

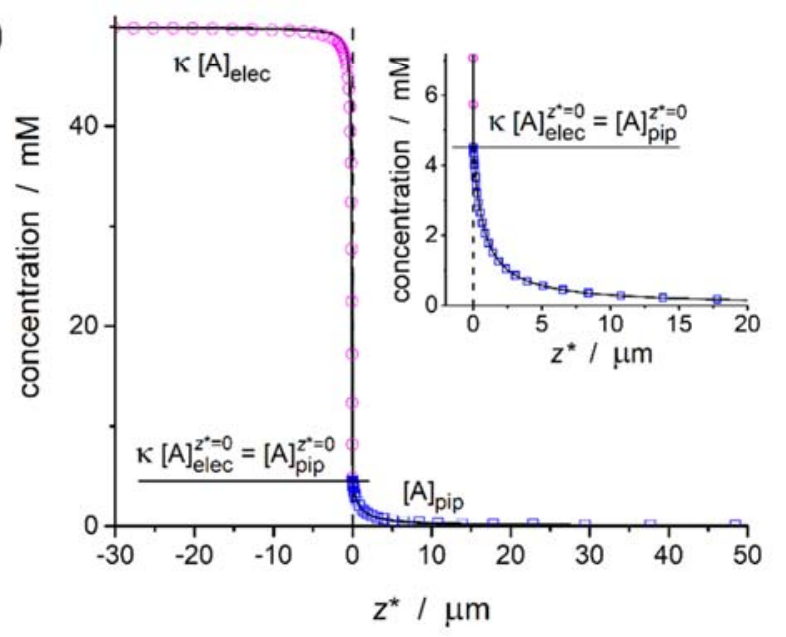

Figure 2. (a) Concentration gradients of species $A$ in the two phases before (left panel) and after (right panel) equilibration when the nanopipette is positioned far from the microdisk electrode; (b) same as (a) with a zoom of the concentration maps shown in (a) in the vicinity of the interface; (c) simulated concentration profile for the species $A$ (black line) along the pipette symmetry axis $(r=0)$ as extracted from the concentration maps shown in the right panels in (a) and (b); $z^{*}$ is the distance from the liquidliquid interface (located at $z^{*}=0$ as featured by the vertical dashed line, see also Fig.1) inside the nanopipette $\left(z^{*}>0\right)$ or in the outer solution $\left(z^{*}<0\right)$; blue squares and magenta circles are the analytical solutions given by Eq.(3) and Eq.(4) in each phase (see text); the inset in (c) shows an expanded view of the concentration profile within the nanopipette. $[A]_{\mathrm{pip}}^{\text {bulk }}=0$ (this value was selected since it is the most difficult computational one with respect to initial conditions), $[A]_{\mathrm{elec}}^{\mathrm{bulk}}=1 \mathrm{mM}, \kappa=50, D_{\text {elec }}^{A}=5 \times 10^{-6}$ $\mathrm{cm}^{2} / \mathrm{s}, \gamma=D_{\text {solv }}^{A} / D_{\text {elec }}^{A}=2, R_{\text {pip }}=100 \mathrm{~nm}, R G=1.5, \theta=8^{\circ}$. 


\section{Simulation results and discussion}

In the following simulations, the separation distance, $d$, between the nanopipette tip and the microelectrode is comparable to $R_{\text {pip }}$, the pipette is moving along the symmetry axis of the microelectrode. At long distances, viz., when $d \gg R_{\text {pip }}$, the nanopipette does not significantly perturbs the diffusion layer at the microelectrode (see insets in Figs.4b and 4c) and may, therefore, be used to map concentration profiles of ionic species created by the microelectrode. Although not immediately relevant to the present work, this approach is complementary to the amperometric or potentiometric monitoring of diffusion layers ${ }^{[14,15]}$ and useful for probing concentration profiles of electrochemically inactive ionic species.

Conversely, when $d$ and $R_{\text {pip }}$ are comparable, the processes occurring at the microelectrode and nanopipette interfere due to their electrochemical properties as well as due to the fact that the nanopipette presence partially screens the diffusional access to the microelectrode surface. For example, in the absence of nanopipette delivery (viz., when the electroactive species, $A$, is initially present only in the bulk electrolyte solution, i.e., $[A]_{\mathrm{elec}}^{\text {bulk }} \neq 0$ and $[A]_{\mathrm{pip}}^{\text {bulk }}=0$ ), one observes (Figure 3 , black curve) a behavior reminiscent of that of a negative feedback in scanning electrochemical microscopy (SECM). Indeed, when $d / R_{\text {pip }}$ is less than a few units, the shaft of the nanopipette prevents diffusional access to the microelectrode area facing the liquid-liquid interface, thus avoiding any significant electrogeneration of the ionic product of the reaction, $B$, over this area. $B$ is still produced around the pipette tip but the nanopipette can collect it only through edge diffusion. This leads to a decrease in the collection efficiency when $d / R_{\text {pip }}$ is decreased. Conversely, when the nanopipette is retracted sufficiently far, one observes the unperturbed $B$ concentration gradient generated by the microelectrode. Note, that in Figure 3 the concentration gradient when $d / R_{\text {pip }}>10$ appears negligible owing to the small span of $d / R_{\text {pip }}$ values reported in this plot compared to the very large relative value of $R_{\text {disk }} / R_{\text {pip }}$ (compare the insets in Figs. $4 \mathrm{~b}$ and 4c shown below).

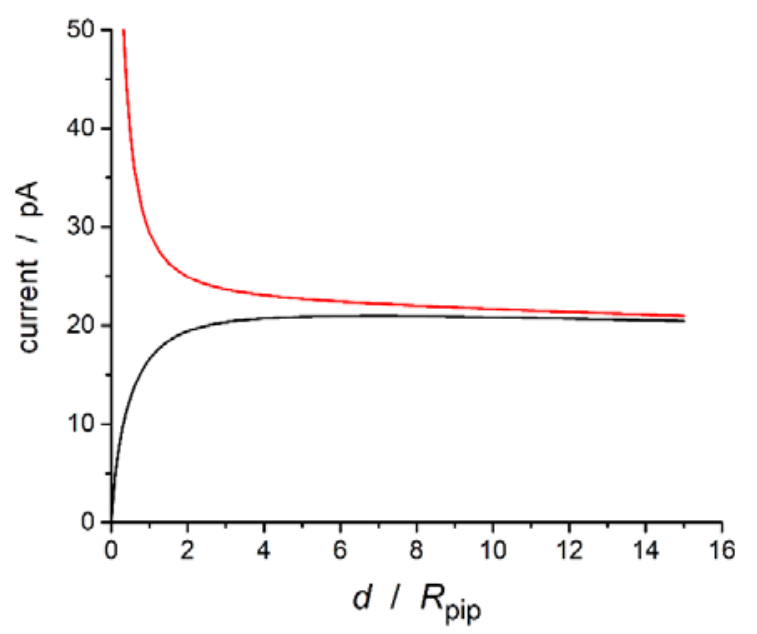

Figure 3. The variation of the simulated pipette current for $[A]_{\mathrm{elec}}^{\text {bulk }}=1 \mathrm{mM}$ when $[A]_{\mathrm{pip}}^{\text {bulk }}=0$ (black curve) and $[A]_{\text {pip }}^{\text {bulk }}=50 \mathrm{mM}$ (red curve). Other conditions are the same as in Fig.2. 
The red curve in Figure 3 represents the outcome of the same experiment, when $[A]_{\text {pip }}^{\text {bulk }} \neq 0$, viz., when $A$ is present in the electrolyte bulk and simultaneously delivered by the nanopipette. As established in our previous report ${ }^{[1]}$, when $d / R_{\text {pip }}$ is less than a very few units, the nanopipette-electrode behaves as a thin layer cell. Hence the current contribution due to the nanopipette delivery tends to vary as $R_{\mathrm{pip}} / d$, thus grows to infinity when $d / R_{\text {pip }} \rightarrow 0$. The corresponding sharp current increase due to the nanopipette local delivery may largely compensate or not the decrease observed when $[A]_{\text {pip }}^{\text {bulk }}=0$ (black curve in Figure 3 ) depending on the ratio $[A]_{\text {pip }}^{\text {bulk }} /[A]_{\text {elec }}^{\text {bulk }}$ as well as on the partition coefficient, $\kappa$, of $A$ between the two liquids. This is evidenced in Figure $4 a$ through a comparison of these individual contributions, i.e., upon considering a nanopipette delivery alone (i.e., $[A]_{\mathrm{elec}}^{\mathrm{bulk}}=0$, and different $[A]_{\mathrm{pip}}^{\mathrm{bulk}}$ values, green curves) or an initial presence of $A$ only in the bulk electrolyte (i.e., $[A]_{\text {pip }}^{\text {bulk }}=0$, and different $[A]_{\text {elec }}^{\text {bulk values, blue }}$ curves). This series of plots evidences that when both contributions coexist simultaneously one may observe two distinct behaviors depending on the relative values of $\kappa$ and $[A]_{\text {pip }}^{\text {bulk }} /[A]_{\text {elec }}^{\text {bulk }}$. One is reminiscent of the red curve in Figure 3 , viz., in which the ionic pipette currents decays monotonously while $d / R_{\text {pip }}$ increases (Figure $4 \mathrm{~b}$ ). The other behavior is illustrated in Figure $4 \mathrm{c}$ and is observed when $\kappa$ and $[A]_{\mathrm{pip}}^{\mathrm{bulk}} /[A]_{\mathrm{elec}}^{\mathrm{bulk}}$ are small enough for the thin layer cell contribution due to the nanopipette delivery results too small to compensate for the screening of the microelectrode activity by the nanopipette shaft. This is an interesting feature that provides an experimental access to a simple in situ measurement of $\kappa$ based on diffusion kinetics rather than on classical methods based on equilibrium between different phases. ${ }^{[16]}$ For example, as shown in Figure $4 \mathrm{~d}$, for any given $\kappa$ value and an appropriate value for $[A]_{\text {elec }}^{\text {bulk }}$, modifying $[A]_{\text {pip }}^{\text {bulk }}$ allows recording a progressive transition from one type of behavior to the other one that is characteristic of $\kappa$, which could then be extracted by fitting the simulated approach curves to one or few experimental ones. The generality of this behavior for a range of $\kappa$ values is demonstrated in Supporting Information. In this respect it is emphasized that even if the red curves in Figures $4 \mathrm{~b}$ and $4 \mathrm{c}$ were simulated under conditions where species $A$ was present in both phases, they represent the exact summation of the individual curves simulated when $A$ is present only in one of the two phases. This property provides a very simple access to the construction of ensembles of approach curves as those shown in Figure $4 \mathrm{~d}$ for a wide range of situations.

It should also be noted that, though original, this approach takes advantage of the same diffusional competition that can be monitored through voltammetric measurements (see e.g. ref. [17]). However, its main advantage is that it relies on the steady state ionic currents easily attained at nanointerfaces thus making analysis more precise and robust. 

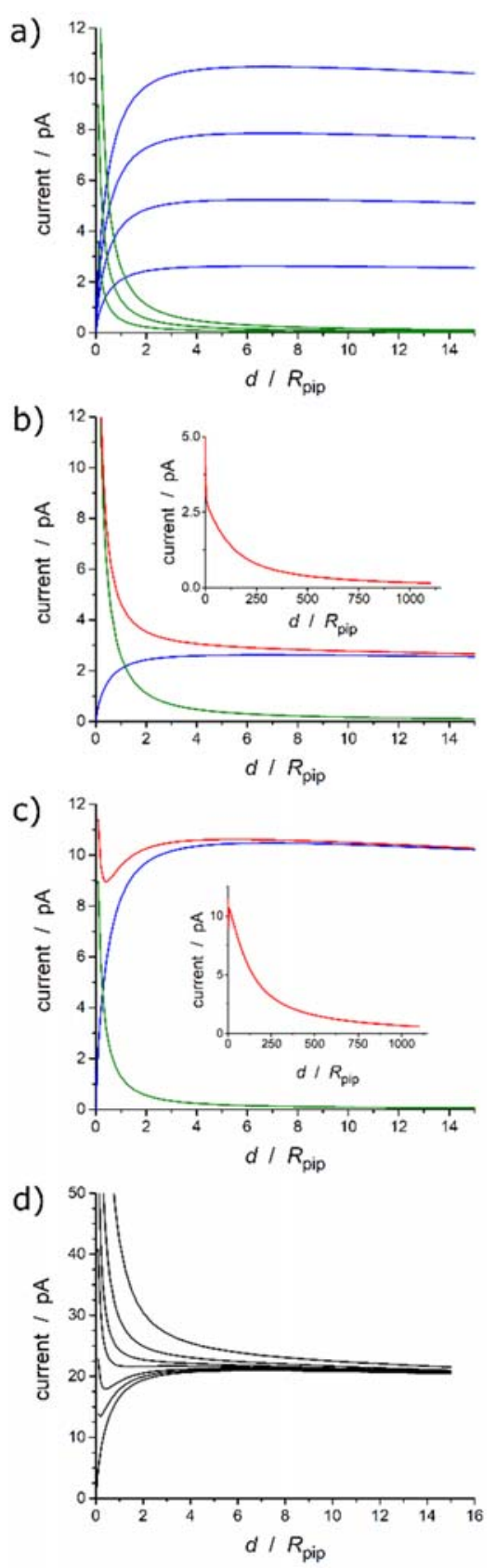

Figure 4. (a) Approach curves for the case when $[A]_{\mathrm{elec}}^{\text {bulk }}=0$ (green lines) for $[A]_{\mathrm{pip}}^{\text {bulk }}=10,5,2 \mathrm{mM}$ (from top to bottom) and $[A]_{\text {pip }}^{\text {bulk }}=0$ (blue lines) for $[A]_{\text {elec }}^{\text {bulk }}=0.5,0.375,0.25,0.125 \mathrm{mM}$ (from top to bottom); (b) red approach curve was simulated for $[A]_{\text {pip }}^{\text {bulk }}=10 \mathrm{mM}$ and $[A]_{\text {elec }}^{\text {bulk }}=0.125 \mathrm{mM}$; (c) red approach curve was simulated for $[A]_{\text {pip }}^{\text {bulk }}=5 \mathrm{mM}$ and $[A]_{\mathrm{elec}}^{\text {bulk }}=0.5 \mathrm{mM}$; (d) approach curves for $[A]_{\text {elec }}^{\text {bulk }}=1 \mathrm{mM}$ and different $[A]_{\text {pip }}^{\text {bulk }}=0,5,10,20,30,50,100 \mathrm{mM}$ (from bottom to top). In (b) and (c) green and blue curves are identical to those shown in (a) for the corresponding values of $[A]_{\text {pip }}^{\text {bulk }}$ and $[A]_{\text {elec }}^{\text {bulk; }}$ the insets provide the variations of the red approach curve at long distances thus featuring the diffusional decay of product B (see text). Other conditions are the same as for Fig.2. 

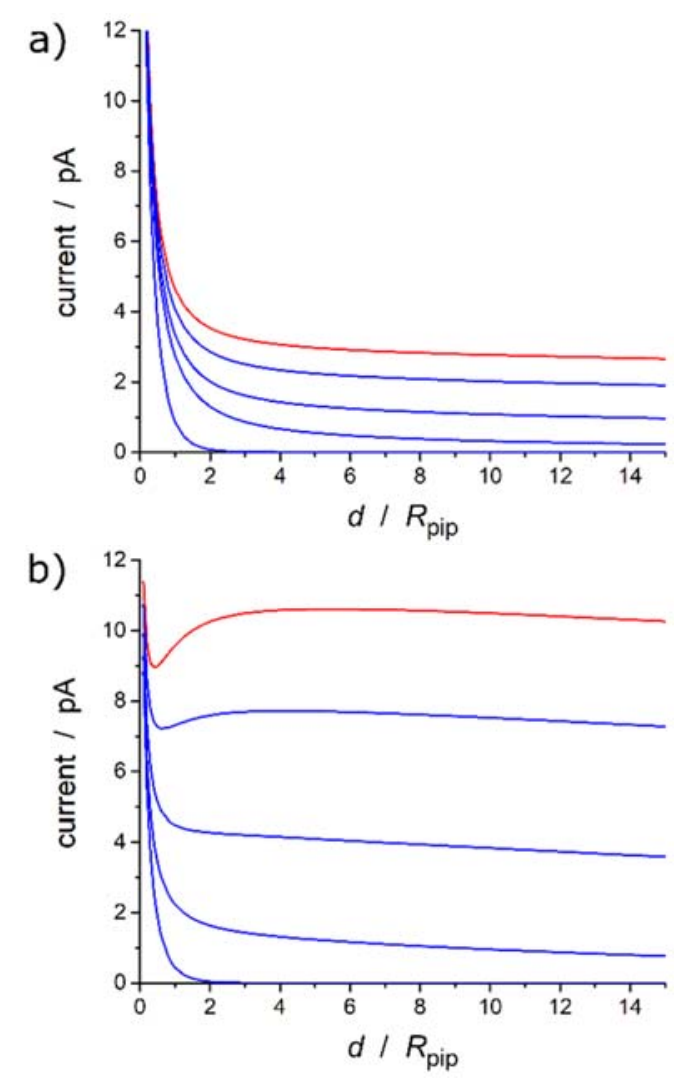

Figure 5. The approach curves obtained for (a) $[A]_{\text {pip }}^{\text {bulk }}=10 \mathrm{mM},[A]_{\mathrm{elec}}^{\text {bulk }}=0.125 \mathrm{mM}$ and (b) $[A]_{\text {pip }}^{\text {bulk }}=$ $5 \mathrm{mM},[A]_{\text {elec }}^{\text {bulk }}=0.5 \mathrm{mM}$ in the absence of homogenous reactions (red lines, as shown in Figs. $4 \mathrm{~b}$ and $4 \mathrm{c}$ also in red) or in presence of the first order following homogeneous reaction (blue lines) with $k_{1}=$ $1,10,10^{2}, 10^{5} \mathrm{~s}^{-1}$ (from top to bottom). Other conditions same as in Fig.2.

The above results correspond to situations in which $B$, the ionic product electrogenerated at the microelectrode, is chemically stable. Let us now examine the influence of homogeneous kinetics. In a preceding work $^{[1]}$ we stressed the nanopipette suitability for detecting extremely short-lived electrogenerated intermediates (life time down to few tens of nanoseconds) when the electroactive substrate $A$ was initially only present in the nanopipette. Unfortunately, in this case the methodology was rather insensitive to slower kinetics. This can be remediated when the electroactive substrate is present in the nanopipette and in the electrolyte solution, as shown in Figures 5 and 6 for first and second order kinetics respectively. These results evidence that the approach curves in these figures are concretely affected over a wide range of first or second order kinetics, hence the corresponding rate constant values could be extracted by fitting simulations to experiment. It is noted that the presence of significant amount of electroactive species $A$ in the outer solution makes the corresponding kinetic term of the diffusionkinetic equation much larger when compared to the case when $A$ is only delivered from the nanopipette. ${ }^{[1]}$ This significantly affects the concentration pattern in the tip-substrate cleft and hence makes the nanopipette current much more sensitive to slow kinetics. Indeed, species $B$ formed outside the thin-layer 
cell and diffusing into the nanopipette have to be transported over distances comparable to the microelectrode radius, $R_{\text {disk }}$ that is much larger than $R_{\text {pip }}$. Hence, slow kinetics may play a significant role by delimiting the dimensions of the portion of the microelectrode from which $B$ can diffuse to the nanopipette orifice.
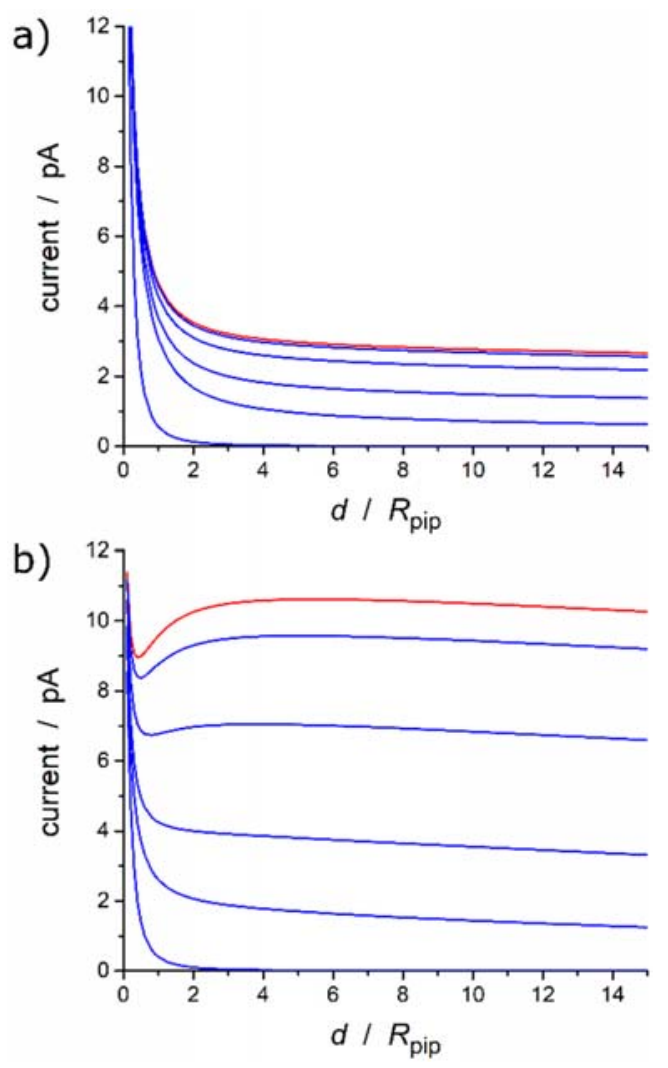

Figure 6. The approach curves obtained for (a) $[A]_{\text {pip }}^{\text {bulk }}=10 \mathrm{mM},[A]_{\mathrm{elec}}^{\text {bulk }}=0.125 \mathrm{mM}$ and (b) $[A]_{\text {pip }}^{\text {bulk }}=$ $5 \mathrm{mM},[A]_{\text {elec }}^{\text {bulk }}=0.5 \mathrm{mM}$ in the absence of any homogenous reactions (red lines, as shown in Figs. $4 \mathrm{~b}$ and $4 \mathrm{c}$ also in red) or in presence of second order follow up homogeneous reaction (blue lines) for $k_{2}=$ $10^{3}, 10^{4}, 10^{5}, 10^{6}, 10^{10} \mathrm{M}^{-1} \mathrm{~s}^{-1}$ (from top to bottom). Other conditions same as in Fig.2.

\section{Conclusions}

This work expanded the concept of using a nanopipette-supported liquid-liquid nanointerface as a probe in the electron transfer/ion transfer SECM mode through investigating the changes introduced when the electroactive species is simultaneously present inside the nanopipette and in the external solution. The system response was evaluated in terms of tip-substrate approach curves under various conditions. This led to two main conclusions in view of potential experimental applications of this alternative operating mode. First, the system response revealed an excellent sensitivity for measurements of partition coefficients across the electrified liquid-liquid nanointerface, suggesting a powerful alternative to classical equilibrium methods, in particular for biological systems. Second, adjusting the relative bulk 
concentrations in the inner pipette solutions and outer liquid phase allows one to extend the capacity of the method to measure the kinetics of homogeneous reactions whose rate constants cover the whole

range of experimental interest, viz., from a few nanoseconds up to a second, under steady-state conditions.

\section{Acknowledgement}

In Paris, this work was supported in part by the Ecole Normale Supérieure, PSL University, Sorbonne University and CNRS (UMR 8640) as well as by LIA CNRS NanoBioCatEchem. Both teams greatly acknowledge the support by the ANR-NSF bilateral (USA-France) program (ANR grant \#ANR-AAP-CE06 "ChemCatNanoTech"; NSF grant CHE-1416116). CA gratefully acknowledges a support from Xiamen University (Xiamen, China) through his Distinguished Professor position. 


\section{References:}

[1] A. Oleinick, Y. Yu, I. Svir, M. Mirkin, C. Amatore, ChemElectroChem 2017, 4, 287-295.

[2] M. Zhou, Y. Yu, K. Hu, M. V. Mirkin, J. Am. Chem. Soc. 2015, 137, 6517-6523.

[3] C. Amatore, C. Lefrou, J. Electroanal. Chem. 1992, 324, 33-58.

[4] C. Amatore, E. Maisonhaute, G. Simonneau, J. Electroanal. Chem. 2000, 486, 141-155.

[5] C. Amatore, E. Maisonhaute, Anal. Chem. 2005, 77, 303A-311A.

[6] P. Fortgang, C. Amatore, E. Maisonhaute, B. Schöllhorn, Electrochem. Commun. 2010, 12, 897-900.

[7] C. Amatore, S. Szunerits, L. Thouin, J.-S. Warkocz, J. Electroanal. Chem. 2001, 500, 62-70.

[8] COMSOL Multiphysics Reference Manual, version 5.3a, COMSOL, Inc, www.comsol.com.

[9] I. Svir, V. M. Golovenko, Electrochem. Commun. 2001, 3, 11-15.

[10] C. Amatore, in Physical Electrochemistry: Principles, Methods and Applications (Ed.: I. Rubinstein), Marcel Dekker, New York, 1995, Chapter 4, Electrochemistry at ultramicroelectrodes.

[11] J. Crank, R. M. Furzeland, J. Inst. Maths. Appl. 1977, 20, 355-370.

[12] A. Oleinick, I. Svir, C. Amatore, Electrochem. Commun. 2004, 6, 588-594.

[13] D. Shoup, A. Szabo, J. Electroanal. Chem. 1984, 160, 27-31.

[14] C. Amatore, S. Szunerits, L. Thouin, Electrochem. Commun. 2000, 2, 248-253.

[15] C. Amatore, S. Szunerits, L. Thouin, J.S. Warkocz, Electrochem. Commun. 2000, 2, 353-358.

[16] J. C. Dearden, G. M. Bresnan, Quant. Struct.-Act. Relat. 1988, 7, 133-144.

[17] S. M. Ulmeanu, H. Jensen, G. Bouchard, P.-A. Carrupt, H. H. Girault, Pharm. Res. 2003, 20, 1317-1322. 